\title{
Indoor MIMO Measurements using Polarization at the Mobile
}

\author{
Tim W. C. Brown, Member IEEE
}

\begin{abstract}
Indoor MIMO channel measurements undertaken with spatially separated dipoles are compared with crossed dipoles at $2.4 \mathrm{GHz}$. This essentially analyses how polarization can be exploited at the mobile using practical antennas where polarization diversity is an inherent feature within an angular diversity system. Results are encouragingly comparable between the two scenarios in terms of capacity and diversity order, which indicates that polarization could be exploited within an angular based MIMO antennas that are more compact than spatially separated antennas.
\end{abstract}

\section{Index Terms - MIMO antenna, Polarization diversity}

\section{INTRODUCTION}

$\mathrm{T}$ WO antenna scenarios are analyzed in this paper. Four half wavelength spatially separated dipoles are compared with the same dipoles that are then co-located as far as is practical and are able to attain de-correlation as a combination of angular and polarization diversity [1] as shown in Figure 1 (a), where increasing the angle $\varphi$ to $90^{\circ}$ will decrease the correlation to zero since the two dipoles have zero angular correlation as shown in Figure 1 (b) if it is assumed the antennas have no mutual coupling. Angular correlation effectively correlates the two angular patterns in the same polarization. Therefore if the polarizations of the antennas have any difference, this will provide an inherent polarization de-correlation within the angular de-correlation. When $\varphi$ is at $90^{\circ}$, it has the greatest polarization de-correlation, which contrasts from the polarization diversity of the fading environment also shown in Figure 1 (b).

The aim of the measurements was to co-locate two orthogonal dipole antennas (i.e. with $\varphi$ equal to $90^{\circ}$ ) as far as is practical so that their capacity potential could be compared with that of the spatial case while at the same time exploiting the polarization diversity available as much as possible from the above analysis. Other investigations into polarization diversity have been based on more ideal assumptions or they have only concentrated on directional antennas at the access point or mobile [2][3][4]. This particular investigation will focus on obtaining de-correlated branches at the mobile end with a wide angle of arrival by which omni-directional antenna branches will always apply both angular and polarization diversity due to practicalities of the antennas. This can then be compared

Manuscript received $4^{\text {th }}$ April 2008.

Tim Brown is with the Centre for Communication Systems Research, University of Surrey, Guildford, Surrey, United Kingdom. Email: tim@brown76.name with the conventional spatial techniques to see whether exploiting use of the polarization in the fading environment is of any benefit.

It was assumed that the fading environment has a reasonable cross polar ratio (XPR) and that the vertical and horizontal polarizations are independent though it was not possible to fully characterize this. It is the case, however, that having the antennas orthogonal to each other will cause some branch power ratio (BPR) greater than that of the spatial case, which is a disadvantage to diversity and MIMO. It is possible that this could be avoided using the $\pm 45^{\circ}$ orientation but as was shown in [1] there is not much polarization contribution within the angular contribution to the de-correlation. The orthogonal orientation is therefore the most appropriate means to identify if the polarization can be best exploited.

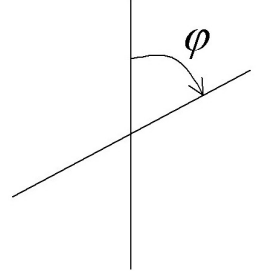

(a)

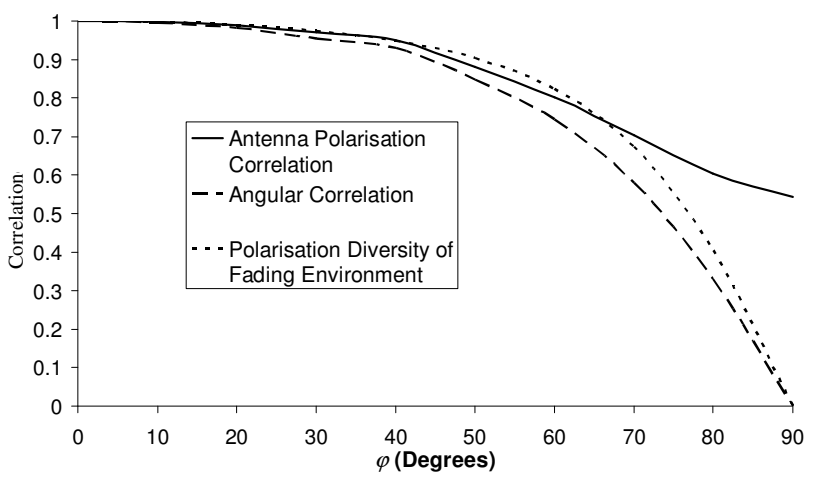

(b)

Figure 1 - Diagram showing the (a) polarization orientation of two dipole antennas and (b) the resultant angular and polarization de-correlation

\section{MEASUREMENT SETUP}

The frequency of operation chosen, due to the scope of the channel sounder used and the available spectrum was $2.4 \mathrm{GHz}$, which is used in WLAN applications. The sampling rate used was $62 \mathrm{~Hz}$, over five times the maximum Doppler shift. There 
was need for such high sampling not just to avoid aliasing but also due to the fact that there were interferences from existing WLANs in the surrounding area that caused occasional disruption to the measurements with bursts of data. In light of this, the data was post processed to remove these intermittent interferences and then replace them with interpolated data. High sampling therefore enabled negligible effect on the resultant impulse responses that were post processed into narrowband fading channels.

The reference dipoles, $0.49 \lambda$ in length are used as the spatially separated case are shown in Figure 2 (a) which are spaced half a wavelength apart for low correlation in the theoretical case. All dipoles have a balun applied to prevent current imbalance. In Figure 2 (b) the dipoles are then moved to a collocated point and crossed. They are located as close as possible while keeping the isolation between them above $10 \mathrm{~dB}$ to try and avoid mutual coupling effects. The horizontal dipole is about $10 \mathrm{~mm}$ behind the vertical one and slightly lower due to the practical problems of the feed cables. In this instance, there is still considerable removal of the spatial case forming a more compact antenna solution so it is largely based upon polarization and angular de-correlation that we get any potential for MIMO.

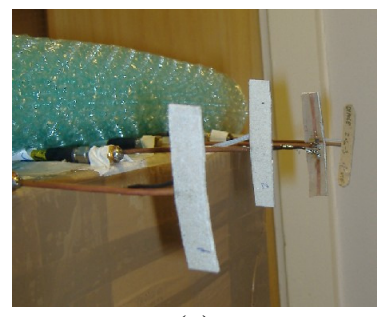

(a)

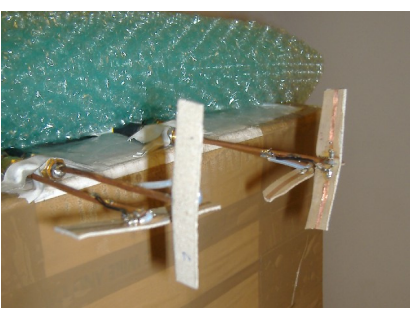

(b)
Figure 2 - Illustrations of the spatial and crossed dipoles used in the measurement

All the antennas tested were measured along the same test run using an Elektrobit Propsound channel sounder. The transmitter was based upon four patch antennas located $2.1 \mathrm{~m}$ high equivalent to that of an access point. The mobile antennas were measured in a neighboring corridor with a non line of sight environment (NLOS) to obtain suitable multipath scattering. It is assumed that both antennas undergo the same radio environment conditions, which is reasonable for the purposes of evaluating channel statistics and not the real time effects or second order statistics.

In order to compare the impact each of the antennas have on scattering and removing path loss effects, it is necessary to carry out a normalization process on the measured channel data. Taking the spatially separated dipoles as the reference case, the path loss is normalized out of the channel, $\mathbf{H}_{\text {spatial }}$, as follows by using the maximum branch magnitude at the mobile end when transmitted from the first transmit (Tx) element. Any other Tx element could be used in this instance as it is assumed that the branch power ratio at the Tx end is unitary. By normalizing this way, it ensures that the branch power ratio at the mobile is preserved, which will have an impact on scattering while removing the path loss isolates any impact it has on the capacity. Thus the channel is normalized as:

$$
\mathbf{H}_{\text {spatial }}{ }^{\prime}=\frac{\mathbf{H}_{\text {spatial }}}{\max \left(\left|h_{\text {spatial } 1 n}\right|\right)}
$$

To evaluate the scattering effects of the crossed dipoles compared to the spaced dipoles, the same normalizing factor used for the spatial case is applied so that when evaluating these two antennas any degradation in gain as well as difference in scattering is taken into account. Thus a fair evaluation is made of the antennas' comparison and two channels for the crossed dipoles, $\mathbf{H}_{P o l}$, are defined as:

$$
\mathbf{H}_{P o l}{ }^{\prime}=\frac{\mathbf{H}_{\text {Pol }}}{\max \left(\left|h_{\text {spatial1 } n}\right|\right)}
$$

Consequently using these normalized channels it is possible to resolve the eigenvalue decomposition, where $\mathbf{S}$ represents the eigenvalues and $\mathbf{V}$ the eigenvectors of a channel $\mathbf{H}$ :

$$
\mathbf{H}^{H} \mathbf{H}=\mathbf{V S V}^{H}
$$

Thus one can calculate the Shannon capacity for a fixed signal to noise ratio (SNR) as follows:

$$
C=\log _{2} \mid \mathbf{I}+\text { SNR.S } \mid
$$

\section{MEASUREMENT RESUlTS}

From measuring the spatial and polarization cases, the correlation was found to be sufficiently low in both cases, below 0.5, which is sufficient for diversity and also multiplexing. For the branch power ratios, it would be expected that the crossed dipoles would be worse than the spatial case, though in these measurements this was only as much as $3 \mathrm{~dB}$ when the ratio between a vertical and horizontal dipole and two vertical dipoles were compared. This suggests there was good de-polarization in the fading environment giving a good XPR though it should be noted the antennas are not polarization pure so the horizontal dipole will still pick up some of the vertical polarization of the fading environment, which is in fact an advantage to balance the branch power ratios better. In the spatial case, the branch power ratio was around $0 \mathrm{~dB}$ between all branches as would be expected.

In terms of the diversity and MIMO capabilities of the spatial and crossed dipoles, their eigenvalues, $s_{1}$ and $s_{2}$, are compared in Figure 3 for 2x2 MIMO whereby just two antenna elements that are spaced half a wavelength are taken from the spatial case at the receive end and two elements from the transmit end. Two crossed dipoles are then compared. Therefore a direct comparison can be made between the spatially separated and differently polarized antennas. The results in Figure 3 show a good comparison in diversity order with comparable eigenvalue distributions. 
It is also possible to make a direct $4 \times 4$ MIMO comparison, whereby all elements from both ends are decomposed into eigenvalues, $s_{1}$ to $s_{4}$. In this instance, a mainly spatial diversity case is being compared with a combined spatial, polarization and angular diversity case and again there are comparable eigenvalues showing similar diversity order in Figure 4. However, in this instance, the first two eigenvalues are shown to be slightly weaker in the polarization case, which is likely to be due to the loss of energy from the horizontal dipoles.

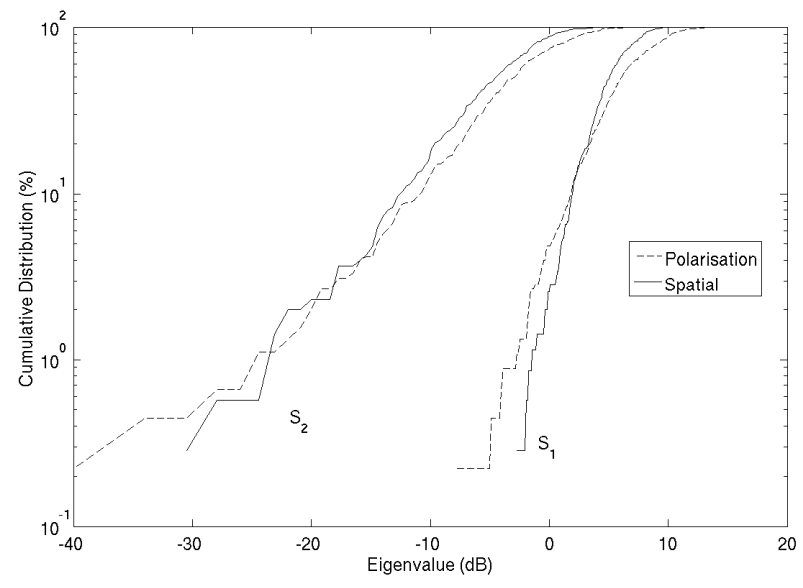

Figure 3 - Direct comparison of the $2 \times 2$ spatial and polarization eigenvalues

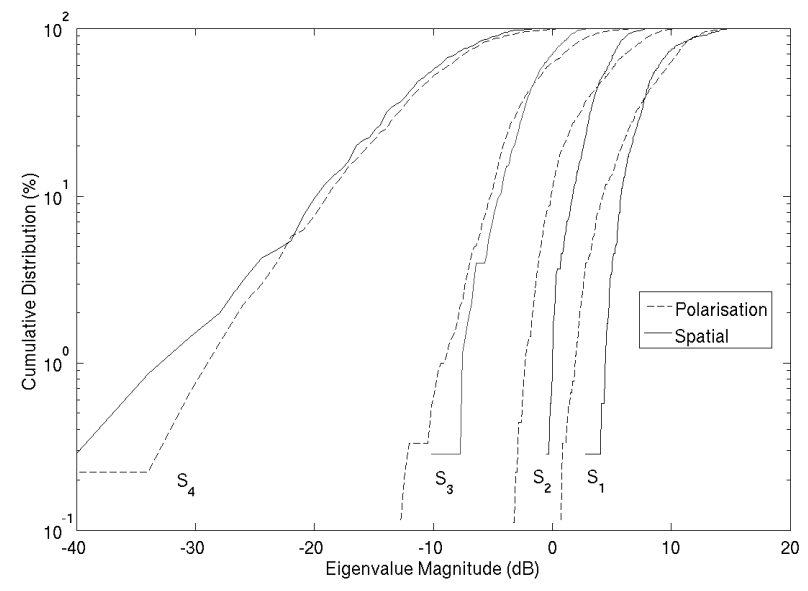

Figure 4 - Direct comparison of the $4 \times 4$ spatial and polarization eigenvalues

Finally the results presented in Figure 5 compare the Shannon capacity for both the $2 \times 2$ and $4 \times 4$ results. In both cases the signal to noise ratio is kept constant at $10 \mathrm{~dB}$ and it is found that comparable capacity limits are shown in the two cases. An unknown that could not be easily characterized is the XPR of the indoor radio environment. This particular information would be a useful indicator to determine whether there is sufficient de-polarization that can be exploited using such antennas for MIMO though the results suggest there is a significant amount. Different environments may not give such complete de-polarization, which could more significantly degrade the MIMO performance since the horizontal dipoles depend largely on the level of de-polarization in the fading environment, though indoor environments can typically offer such requirements.

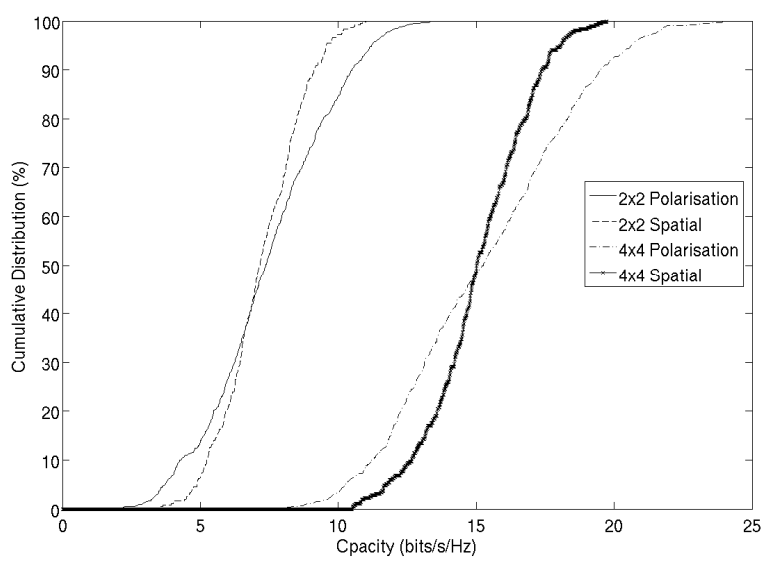

Figure 5 - Direct comparison of the Shannon capacity in both the $2 \times 2$ and $4 \times 4$ spatial and polarization cases

\section{CONCLUSION}

Measurement results comparing mainly spatial with angular and polarization diversity based antennas do indicate that there is potential in using orthogonal linear antennas based on these combinations for MIMO applications, where antenna design possibilities have been analyzed [5][6]. This can allow compact mobile terminals to implement such antennas whereby they use polarization inherently within the angular system to create multiplexing and diversity order. There are, however, further factors to consider on real mobile terminals such as variable antenna orientation and user interaction, where such antennas may not be as effective as using spatial multiplexing. Many mobile terminals, however, are often kept stationary, which does allow scope for using polarizations if the mobile has a normal orientation for usage.

\section{REFERENCES}

[1] T. W. C. Brown, S. R. Saunders, S. Stavrou and M. Fiacco, "Characterisation of Polarization Diversity at the Mobile", IEEE Transactions on Vehicular Technology, vol. 56, issue 5, part 1, pp24402447, September 2007.

[2] J. P. Kermoal, L. Schumacher, F. Frederiksen, P. E. Mogensen, "Polarization diversity in MIMO radio channels: experimental validation of a stochastic model and performance assessment", IEEE Vehicular Technology Conference, vol. 1 pp22-26, Fall 2001.

[3] V. R. Anreddy, M. A. Ingram, "Capacity of measured ricean and rayleigh indoor MIMO channels at $2.4 \mathrm{GHz}$ with polarization and spatial diversity", Wireless Communications and Networking Conference, vol. 2, pp 946-951, 2006.

[4] M. C. Mtumbuka, D. J. Edwards, "Investigation of tri-polarised MIMO technique", Electronics Letters, vol. 41, issue 3, February 2005.

[5] C. Waldschmidt, C. Kuhnert, S. Schulteis, W. Wiesbeck, "Compact MIMO-arrays based on polarisation-diversity", IEEE Antennas and Propagation Society International Symposium, vol. 2, pp449-502, June 2003.

[6] B. N. Getu, J. B. Andersen, "The MIMO cube - a compact MIMO antenna", IEEE Transactions on Wireless Communications, vol. 4, issue 3, pp1136p1141, May 2005. 